\title{
Incorporating Metal Organic Frameworks within Microstructured Optical Fibers towards Scalable Photoreactors
}

Matthew E. Potter, Daniel J. Stewart, Konstantin Ignatyev, Tom Bradley, Pier J. A. Sazio and Robert Raja

M. E. Potter, D. J. Stewart and R. Raja

Department of Chemistry, University of Southampton, Highfield Campus, Southampton, Hampshire, SO17 1BJ, UK

E-mail: M.E.Potter@ soton.ac.uk

K. Ignatyev

Diamond Light Source, Harwell, Didcot, Oxfordshire, OX11 0DE, UK

T. Bradley and P. J. A. Sazio

Optoelectronics Research Centre, University of Southampton, Highfield Campus, Southampton, Hampshire, SO17 1BJ, UK

Keywords: Photonics, Photocatalysis, Photoreactor, Porous Materials, Sustainable Technology.

\begin{abstract}
Optical fiber technology has revolutionized the telecommunications industry, though is still under-utilized in chemistry. Optical fibers open many avenues for introducing, and containing, light in chemical reactions, as part of a photoreactor. This work shows, for the first time, a design strategy for incorporating a photocatalytic, nanoporous framework (Co ZIF-67) within the internal capillaries of an optical fiber, in doing so creating an all-in-one, plug-in-and-play photoreactor. This system improves the reactivity of the photocatalyst, relative to the powdered form, for $\mathrm{C}-\mathrm{H}$ activation leading to $\mathrm{C}-\mathrm{C}$ bond formation, a significant process in pharmaceutical and organic synthesis. Performing this reaction using solar energy, and low temperature demonstrates the clear potential for these systems for large scale industrial applications.
\end{abstract}

Text: The development of optical fibre technology has had a seismic effect on modern society, creating strides in medicine, telecommunications and data storage. ${ }^{[1,2,3]}$ While this principally is due to their impressive light propagation properties, the ability to modulate the 
fibres to induce unique physical and quantum properties makes them of special interest to academics. ${ }^{[4]}$ Furthermore, the cane width and motif are readily tuned, giving multiple levels of control. These systems are also excellent candidates for wide scale implementation, as the telecommunications industry is already bifurcating and growing new optical fibre at a rate of $14,000 \mathrm{ft} / \mathrm{sec}^{[5]}$ Despite being well established in so many areas, optical fibre technology has unrealised potential in many other fields, particularly in the design of novel photocatalytic microreactors, for industrial scales.

Photocatalysis requires the design of effective active sites, but also efficient photon transfer, to use light as a sustainable activation source. Previously researchers have used optical fibres by replacing external cladding with titania, allowing waveguided photons to refract through the high index titania, to the external surface. ${ }^{[6,7]}$ However this reduces the efficiency as the photons must be launched into the end of the waveguide in order to propagate through the highly refractive titania. Optical fibres have since evolved beyond single core motifs. A vast array of microstructured optical fibres have since been developed, with multiple cores in different shapes and sizes. ${ }^{[8,9]}$ These have many potential benefits as microfluidic reactors, over single core systems, including improved surface area and limited pressure drop. In our approach we have grown a metal organic framework (MOF) exclusively on the internal surface of the many capillaries within a microstructured optical fibre canes (MOFCs), thus the optical fibre is not just a photon delivery system but is an all-inclusive photocatalytic reactor (Figure 1). Here liquids and gases flow through the optical fibre, where the internal walls are coated with photocatalyst. Photons are pumped orthogonally to the fibre axis, differing from the previous waveguided approach, by using a parabolic trough optical concentrator, which means the photons are trapped within the capillaries of our coated MOFC system. This creates a unique space inside the channels which combines photons, fluids and catalyst directly within the MOFC photoreactor. Such technology has huge potential to be scaled up and out for industrial photocatalytic flow chemistry (Figure S1). 




Figure 1. Schematic showing our unique methodology for creating an all-inclusive photocatalytic microreactor, coating a metal organic framework (Co ZIF-67) within the channels of an optical fibre, and potential applications for $\mathrm{C}-\mathrm{H}$ activation to selectively form C-C bonds.

Titania has for many decades been among the most prolific photocatalysts, owing to its low cost and ease of availability. ${ }^{[10]}$ In our previous work we showed the ability to incorporate titania into similar MOFCs, and decorate the titania with metallic Pd nanoparticles. ${ }^{[11]}$ Modern photocatalysts have since evolved beyond this and now include a wide range of more advanced framework species. ${ }^{[12,13]}$ Metal organic frameworks (MOFs) are excellent examples of this and have been widely used as photocatalysts for a plethora of applications. ${ }^{[14,15]}$ The high density of metal active sites within MOFs, combined with the synthetic methods to tune the precise nature of these metal sites makes them highly desirable as photocatalysts. MOFs and particularly ZIFs (Zeolitic Imidazolate Frameworks) have been incorporated onto various supports including carbon fibres, ${ }^{[16]}$ porous inorganic fibres ${ }^{[17]}$ and capillary columns. ${ }^{[18]}$ However, in this work we show our novel technology can selectively coat the internal channels of an MOFC with a defined nanoporous framework; Co ZIF-67, for the first time. This framework was chosen as its intense purple colour makes it an excellent light harvesting species, with many different synthesis protocols existing that could be readily adapted to incorporate within capillary fibres. ${ }^{[19,20]}$ We then demonstrate the efficacy of this technology for photocatalytic flow chemistry (Figure S3), with a cross-dehydrogenative coupling (CDC) reaction, activating two $\mathrm{C}-\mathrm{H}$ bonds to form a $\mathrm{C}-\mathrm{C}$ bond. ${ }^{[21]} \mathrm{We}$ then contrast the photocatalytic activity of our Co ZIF-67, both coated on the interior of an optical fibre, with 
the powdered equivalent. Herein we discuss our design strategy, confirm the integrity of the system and finally demonstrate its effectiveness for this chemical transformation.

The MOFCs chosen were $1 / 8$ " in diameter, with over 200 capillaries tessellated with hexagonal and triangular channels, arranged in a Kagome lattice (Figures S4 and S5). While a range of procedures exist for modulating the external surface of such species, or for producing thin films, few can selectively activate the internal surfaces. This makes growing MOF and ZIF species directly onto these surfaces highly challenging. As such we initially introduce a 'binding layer' of P25 titania to the internal capillaries, from which photocatalytic ZIF species can be grown (Figure S2). We note that, despite our best efforts, we were unable to grow the ZIF directly onto the fibre, hence the need for the titania binding layer. It is likely the surface defects of the $\mathrm{P} 25 \mathrm{TiO}_{2}$, and the more ionic nature of the titania, aided the binding of the $\mathrm{ZIF}$ reagents, helping it to adhere to the fibre. The titania was deposited using a dilute suspension of $0.1 \mathrm{wt} \% \mathrm{P}_{2} 5 \mathrm{TiO}_{2}$ in water, then calcined in air at $400{ }^{\circ} \mathrm{C}$ to anneal the titania to the cane. The more dilute titania solution achieved a more even coating, with fewer particulate build ups, as shown by synchrotron microfocus X-ray fluorescence (XRF) mapping at $9 \mathrm{keV}$ incident photon energy (Figure 2 and S5). When focussing on the Ti emissions at $4.51 \mathrm{keV}$ (Ti $\mathrm{K}_{1} / \alpha_{2}$, Figure $2 \mathrm{~A}$ ), titania can clearly be seen along the walls of the internal capillaries, and not as bulky particles, suggesting a comparatively smooth coating. ICP analysis estimates the total amount of titanium in this system to be incredibly low at roughly $0.01 \mathrm{wt} \%$ (Table S1).

The Co ZIF-67 coating was then added by modifying a procedure by Qian et al, ${ }^{[19]}$ known to rapidly make nanoscale Co ZIF-67 particles of sizes between 100-400 nm. As ZIF synthesis is highly sensitive to the synthetic conditions used, it may be possible to control particle size, shape and loading of the ZIF onto the cane. This confers great synthetic control to the researcher, however for the purpose of this proof-of-concept study, only one synthetic protocol was followed. The titania coated canes were stirred in an aqueous mixture of cobalt nitrate hexahydrate and 2-methyl imidazole. Attempts to incorporate Co ZIF-67 on canes without a titania layer were unsuccessful, clearly illustrating that the ZIF only adhered to the internal titania layers. On drying the canes were sonicated in ethanol to remove residual particles and dried again. This process was performed three times, after which a clear purple coating was seen exclusively within the canes (Figure 3C). Particles formed from this process that were not adhered to the cane were also collected, and confirmed as Co ZIF-67 via powder XRD, $\mathrm{N}_{2}$ physisorption and ICP analysis (Figure S6). ${ }^{[19,20]}$ In the XRF spectra, the intensity of 
the $\mathrm{Ti} \mathrm{K} \alpha_{1} / \alpha_{2}$ emission lines were relatively small (Figure $2 \mathrm{C}$ ), however intense signals at 6.90 and $7.65 \mathrm{keV}$ were all present, attributed to $\mathrm{Co} K \alpha_{1} / \mathrm{K} \alpha_{2}$ and $\mathrm{K} \beta_{1}$ emission lines respectively (Figure 2B). All XRF spectra containing noticeable Co signals at 6.90 and 7.65 $\mathrm{keV}$ also showed a $\mathrm{Ti}$ signal at $4.55 \mathrm{keV}$, suggesting that $\mathrm{TiO}_{2}$ satisfactorily worked as a binding layer for incorporating Co ZIF-67 into the optical fibre canes. A feature at $2.96 \mathrm{keV}$ can also be seen, which is attributed to argon contained in the air.
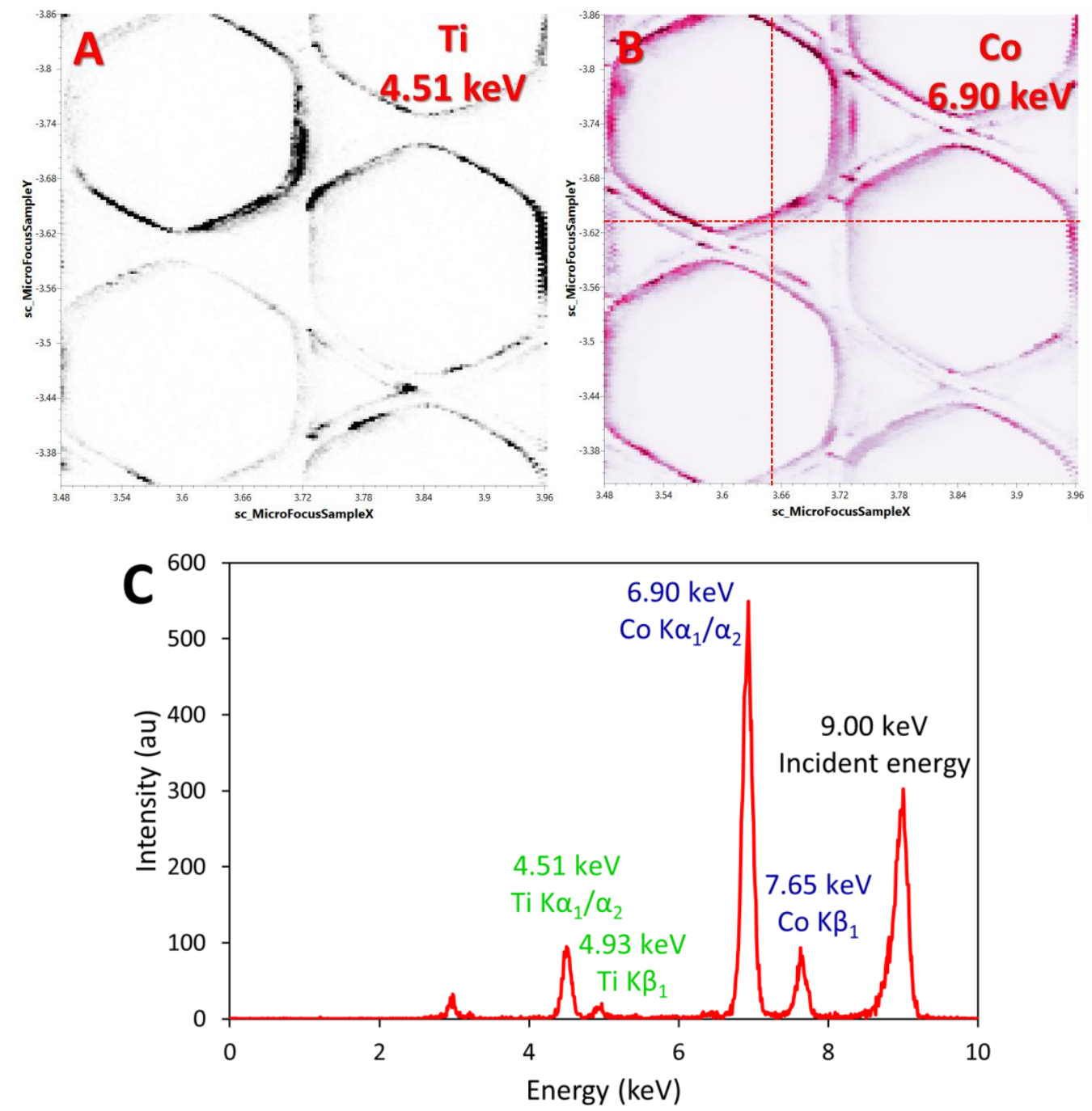

Figure 2. X-ray Fluorescence maps of a hexagonal capillary wall of a Co ZIF-67 coated fibre, highlighting emissions from A) Ti $\mathrm{K} \alpha_{1} / \alpha_{2}$ at $4.51 \mathrm{keV}$, B) Co $\mathrm{K} \alpha_{1} / \alpha_{2}$ at $6.90 \mathrm{keV}$. In both cases darker colours refer to higher concentrations. C) Associated X-ray fluorescence spectra from the marked point on Figure 2B. Axes in fluorescence maps are in mm.

Subsequent microfocus X-ray fluorescence imaging, focussing on emissions at $6.90 \mathrm{keV}$ also showed coating of cobalt, due to Co ZIF-67, over all the internal capillaries of the canes (Figure 2B). ICP analysis showed that the total amount of cobalt present in the cane was 0.07 
wt\% (Table S1). The structural integrity of the Co ZIF-67 species was confirmed using microscopy images combined with Raman spectroscopy (Figures 3 and S7). The microscopy images instantly show a stark difference in colour on coating with Co ZIF-67, as seen by contrasting Figures S7A and S7C. In the $\mathrm{TiO}_{2}$ coted cane (Figure S7C) little colour is seen, however a purple hue is clearly seen in Figures S7A. Figure S7D clearly shows the deposition of the purple Co ZIF-67 down the length of the fibres interior.
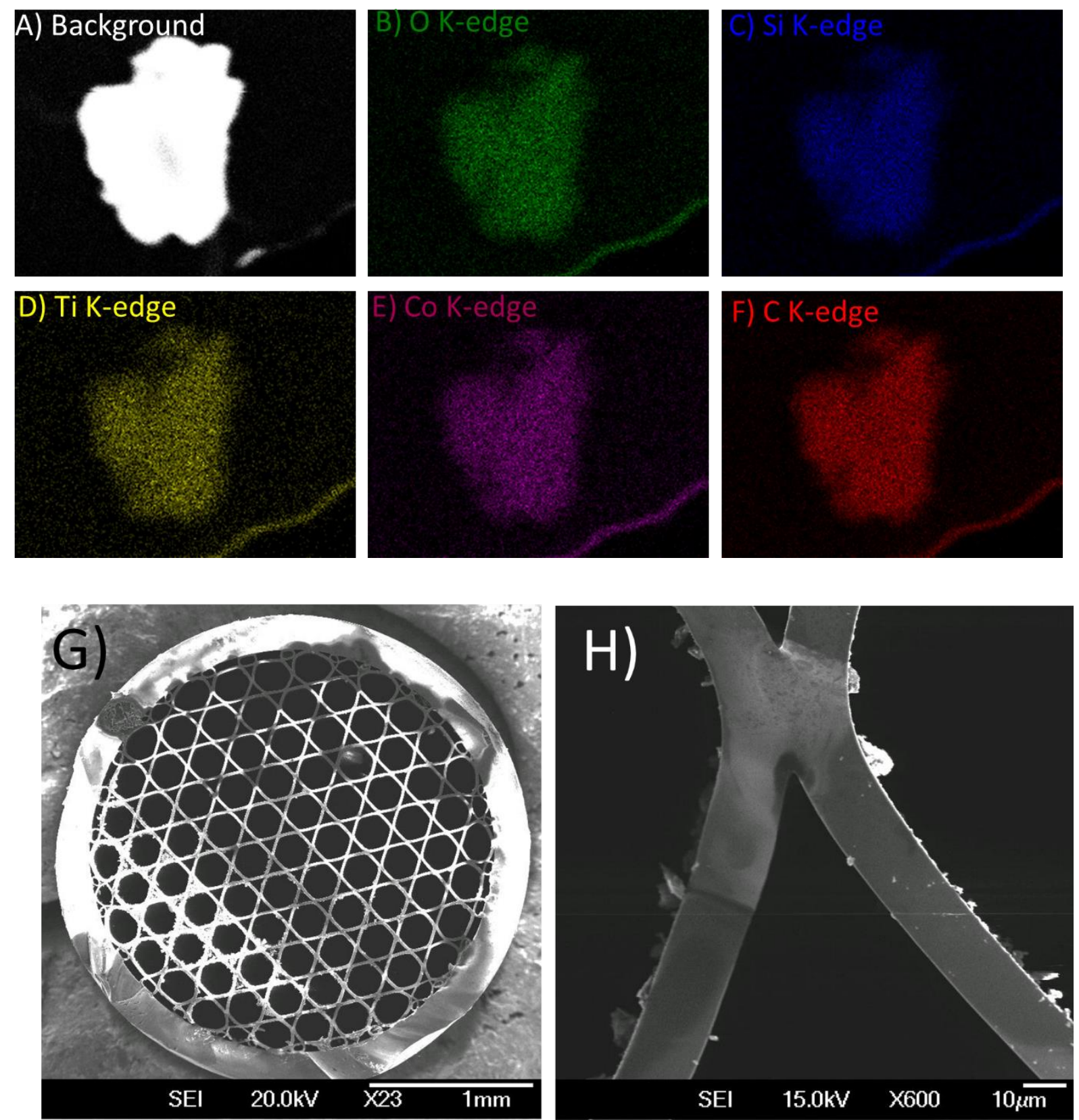

Figure 3: TEM-EDX maps showing the uniform distribution of different elements. A) Shows the bright field image, B) and C) are O and Si respectively, primarily from the bare MOFC cane. D) shows the Ti distribution from the $\mathrm{P} 25 \mathrm{TiO}_{2}$ layer. Finally E) and F) are $\mathrm{Co}$ and $\mathrm{C}$ 
respectively, derived from the Co ZIF-67. G) SEM image of the in-tact Co ZIF-67 coated MOFC. H) SEM image showing the formation of Co ZIF-67 crystals on the interior of the MOFC cane.

Transmission electron microscopy (TEM) was used in combination with Energy Dispersive X-ray (EDX) spectroscopy to probe the distribution of different elements (Figure 3 \& S8). For this work, the MOFC cane was crushed into a fine powder to ensure it could be successfully imaged. Imaging these MOFC systems, without the need for crushing them is still on-going. Figures 3A-F show the TEM image of particles from the cane, and EDX elemental maps of this area, respectively. These images show that all 5 elements ( $\mathrm{O}, \mathrm{Si}, \mathrm{Ti}, \mathrm{Co}$ and $\mathrm{C})$, arising from various stages of the coating process $\left(\mathrm{O} \& \mathrm{Si}\right.$ bare cane, $\mathrm{Ti} \mathrm{TiO}_{2}$ coating and $\mathrm{Co} \& \mathrm{~N}$ Co ZIF-67) are uniformly distributed across this particle, confirming the composition and deposition of these materials on the MOFC.

Scanning electron microscopy (SEM) images, of the cane itself, without disruption, confirmed the adhesion of the Co ZIF-67 to the cane (Figures 3G \& 3H). Looking down the cane a range of crystalline particles can be seen. Focussing on specific areas reveals crystalline particles, roughly 1-10 $\mu \mathrm{m}$, growing off of the MOFC cane, which are of similar size to those seen in our previous work in powdered Co ZIF-67. ${ }^{[22]}$ We note that there is little evidence of P25 $\mathrm{TiO}_{2}$ inclusion on this scale, as nanopowder $(20-30 \mathrm{~nm})$ was used for the coating. The particles are exclusively found inside the MOFC channels and capillaries, confirming their incorporation inside the system.

Multiple points were examined using Raman spectroscopy, with the fused silica optical fibre spectra being primarily featureless in all cases. Points directly adjacent to the capillary walls were in excellent agreement with previous reports for $\mathrm{P}_{2} 5 \mathrm{TiO}_{2}$ (Figure 4A), showing large signals at $143,398,517$ and $640 \mathrm{~cm}^{-1}$ attributed to the $E_{g}, B_{1 g}, A_{1 g} / B_{2 g}$ and $E_{g}$ stretches of the anatase phase, with a small signal present at $197 \mathrm{~cm}^{-1}$, attributed to the rutile phase, as expected for $\mathrm{P} 25 \mathrm{TiO}_{2} \cdot{ }^{[23]}$ Other more subtle features can be seen at higher Raman shifts, which are in good agreement with previous spectra on Co ZIF-67, ${ }^{[24]}$ again showing the proximity of these two layers. The purple layer of the MOFC was also probed (Figure 4A), and aside from features already attributed to titania, showed excellent agreement with 
previously published spectra for bulk Co ZIF-67 (Figure S9). ${ }^{[24]}$ This confirmed the framework had been satisfactorily synthesised within the confines of the internal capillaries of the optical fibre (Figure 1). ${ }^{[24]}$ The spectra were notably noisier than literature spectra of bulk Co ZIF-67, though this is likely due to low loadings of cobalt and the presence of the fused silica and titania phases. However, this combined with the microfocus X-ray fluorescence spectroscopy shows this is the first example of a defined MOF framework being selectively designed and confined within an MOFC.
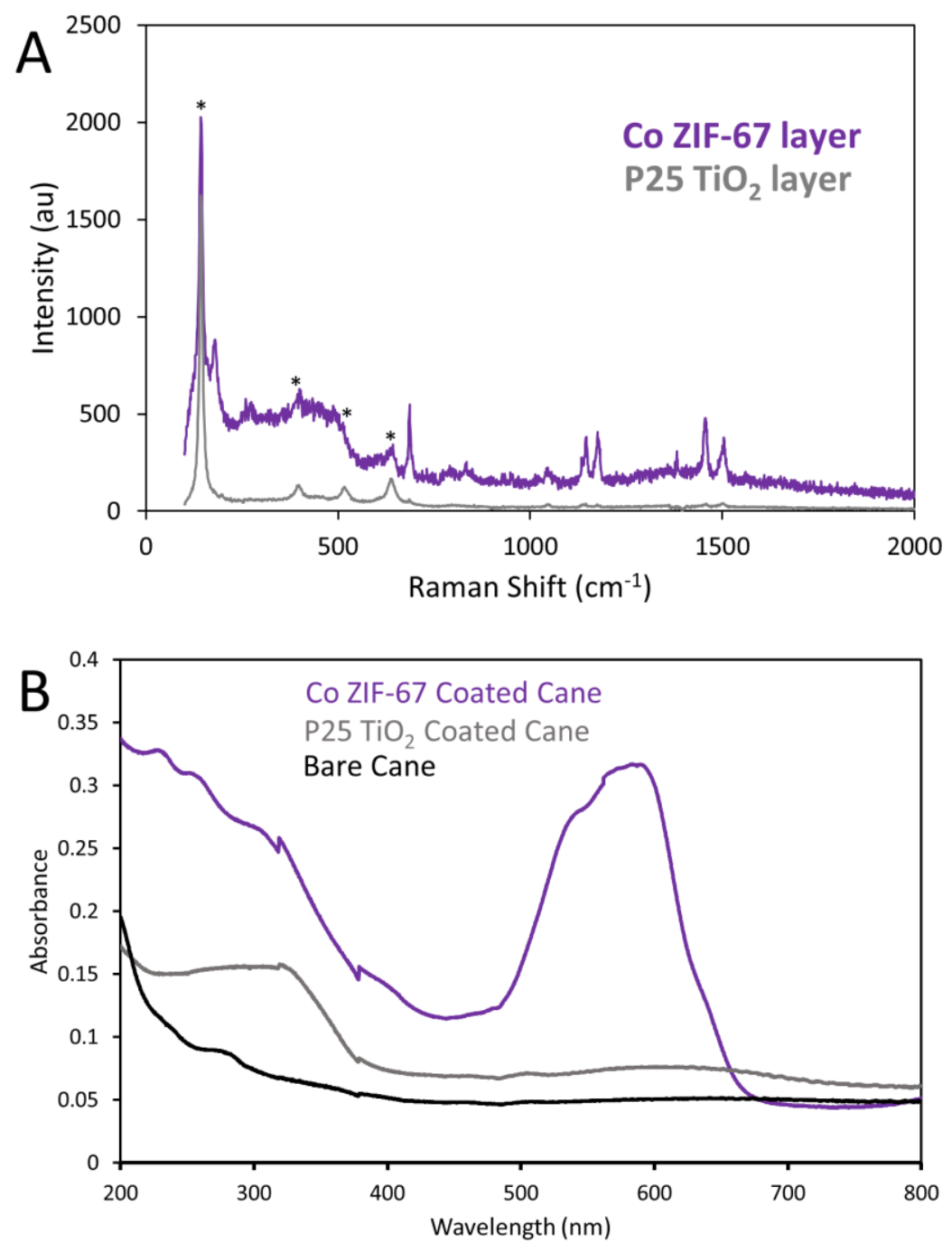

Figure 4. A) Raman spectra highlighting the $\mathrm{ZIF}$ and $\mathrm{TiO}_{2}$ layers, taken from microscope image Figure S5D. * Represent signals due to $\mathrm{TiO}_{2}$ in the Co ZIF-67 spectra. B) UV/Vis spectra comparing the absorption properties of the bare cane, with the same cane coated with P25 $\mathrm{TiO}_{2}$ and then Co ZIF-67. 
Further UV/Vis data was collected on the canes, supporting the incorporation of Co ZIF-67 and $\mathrm{P} 25 \mathrm{TiO}_{2}$ onto the canes (Figure 4B). Contrasting the bare cane with the $\mathrm{P} 25 \mathrm{TiO}_{2}$ coated cane we see increased absorbance at $200 \mathrm{~nm}$, which decreases sharply between 300 and 400 $\mathrm{nm}$. This is in good agreement with previous measurements on $\mathrm{P} 25 \mathrm{TiO}_{2}$ powder, supporting its deposition on the system. ${ }^{[25]}$ On addition of the Co ZIF-67 material, strong features are seen in the $500-600 \mathrm{~nm}$ range, as seen in our previous work on Co ZIF-67 powder. ${ }^{[22]}$ This feature is a combination of three signals, encompassing the ${ }^{4} \mathrm{~A}_{2}\left({ }^{4} \mathrm{~F}\right) \rightarrow{ }^{4} \mathrm{~T}_{1}\left({ }^{4} \mathrm{P}\right),{ }^{4} \mathrm{~A}_{2}\left({ }^{4} \mathrm{~F}\right) \rightarrow$ ${ }^{4} \mathrm{~T}_{1}\left({ }^{4} \mathrm{~F}\right)$ and ${ }^{4} \mathrm{~A}_{2}\left({ }^{4} \mathrm{~F}\right) \rightarrow{ }^{4} \mathrm{~T}_{2}\left({ }^{4} \mathrm{~F}\right) \mathrm{d}-\mathrm{d}$ cobalt(II) transitions. Further new features are also seen around $250-300 \mathrm{~nm}$, which is indicative of a cobalt-oxygen metal-to-ligand charge transfer, commonly seen in cobalt(II) tetrahedral species. These findings, in combination with the Raman spectroscopy (Figure 4A) strongly support the formation of Co ZIF-67 within MOFC. Further, coating the MOFC with such materials increases light absorption in the UV/Vis range, making them suitable solar photocatalysts.

Cobalt-based materials have recently been shown to be active photocatalysts for the AzaHenry CDC reaction ${ }^{[22,26]}$ with N-phenyl-1,2,3,4-tetrahydroisoquinoline (PhTHIQ) under batch conditions. ${ }^{[22,26]}$ We therefore compared the activity of our novel ZIF-coated MOFCs, to powdered Co ZIF-67 under batch conditions for a similar Aza-Henry reaction (Figure 5).

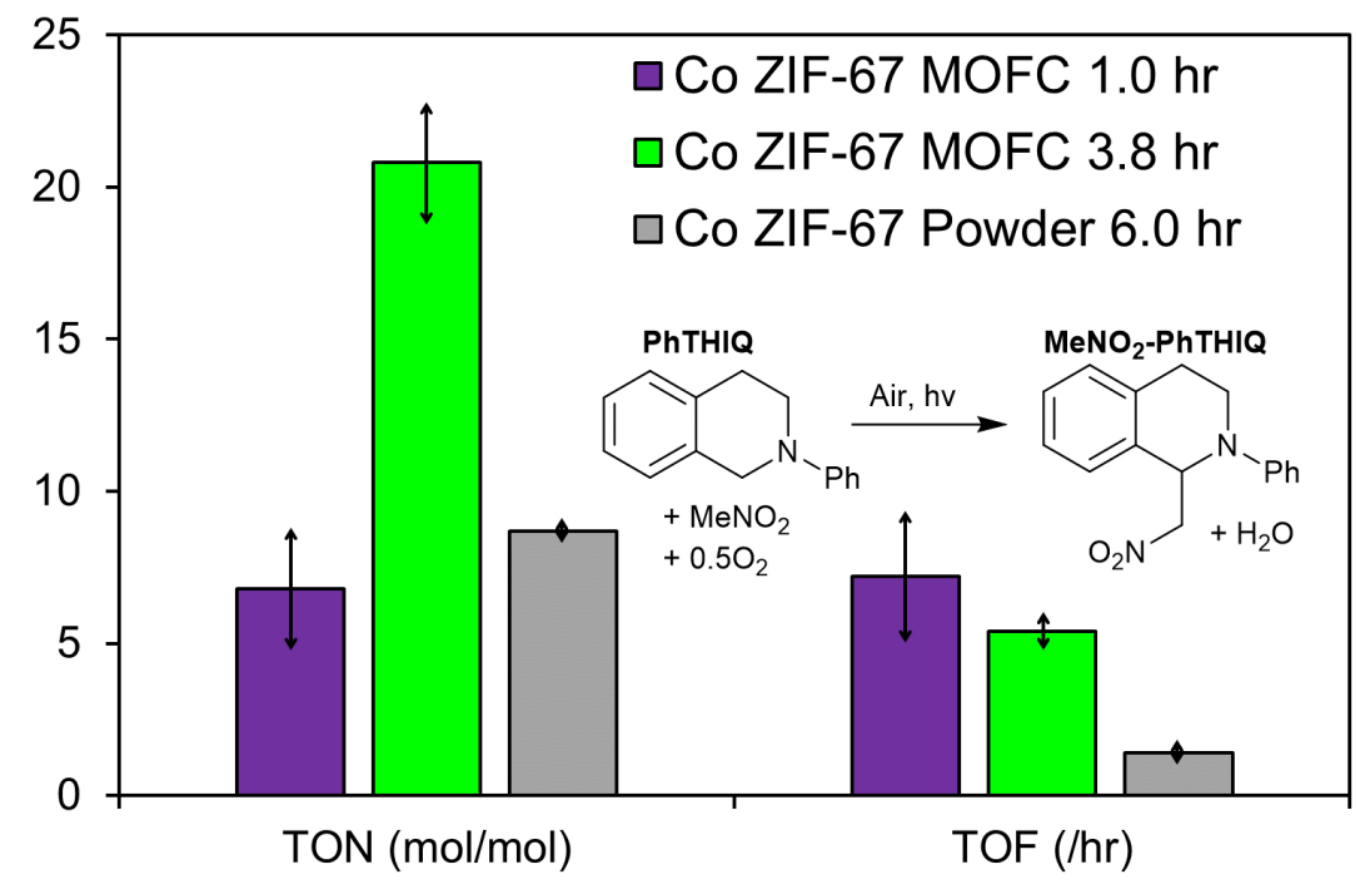

Figure 5. Contrasting the photocatalytic activity for the Aza-Henry CDC reaction using Co ZIF-67, when coated on an optical fibre, as a function of contact time, with analogous 
powder. Co ZIF-67 MOFC conditions: $5 \mathrm{~mL} \mathrm{MeNO}_{2}, 125 \mathrm{mg}$ of PhTHIQ, $67.5 \mu \mathrm{L}$ of mesitylene standard, flowing at $0.36 \mathrm{~mL} / \mathrm{min}$, looped reactant solution. Co ZIF-67 Powder conditions: $0.11 \mathrm{mmol}$ of PhTHIQ, $10 \mathrm{wt} \%$ catalyst, $1 \mathrm{~mL} \mathrm{MeNO}$, mesitylene internal standard. For further details, including error estimation, see the ESI.

Under solar light, Co ZIF-67 MOFCs demonstrate unique potential for photocatalytically activating $\mathrm{C}-\mathrm{H}$ bonds, prompting the selective formation of a $\mathrm{C}-\mathrm{C}$ bond. In the MOFC system, the reaction solution was continuously looped through the cane, in an open system, allowing the oxidant (air) to be replenished. Unlike conventional photocatalytic batch processes, we can ensure the system is only exposed to light whilst inside the MOFC (Figure S3), and thus more readily interacts with the photocatalyst. This allows us to simultaneously tailor the light exposure and contact time with the catalyst. Due to the use of optical fibre supports in our MOFC system, photons are waveguided in situ to the photocatalytic coating. In conventional batch systems they are forced to continually refract and scatter before encountering the catalyst. Whereas our parabolic-trough methodology specifically focuses the light onto the catalyst, providing us far greater control over light propagation in the MOFC system. With further work in this area high levels of control could be achieved to fully optimise the MOFC system. The conversion for the Co ZIF-67 MOFC increases with contact time, (Figure 5), showing the stability of our methodology, and its ability to maintain activity within a continuous system. The estimated quantum yields are similar in magnitude $(0.19 \%$ batch, $0.13 \%$ MOFC, see ESI for details) suggesting minor changes in the MSOF preparation or reaction design could see it surpass the batch system for this particular metric. The turnover number (TON) and turnover frequencies (TOFs) were contrasted with conventional powdered Co ZIF-67 photocatalyst, which operated under batch conditions (Figure 5). This shows that the Co ZIF-67 MOFC system leads to improved catalytic efficiency, outperforming the powder in both TOF and TON, demonstrating the benefits of fibre coating for photoreactor design. Namely the ability to tailor light propagation and photon delivery, while creating a space to effectively combine photons, fluids and photocatalysts. Further we compare the influence of adding the Co ZIF-67 layer to the MOFC cane (Figure S10), showing that despite the large background reaction (from higher energy light ${ }^{[22]}$ ), the addition of the Co ZIF-67 does noticeably improve the reactivity of the system, demonstrating our concept. We believe this improved activity is a direct result of the deposited Co ZIF-67, allowing the system to absorb more light in the UV and visible regions, which can be used for photocatalytic transformations such as the CDC reaction. 
We have demonstrated our unique methodology for incorporating a phase-pure nanoporous MOF; Co ZIF-67, inside the interior capillaries of an optical fibre, for the first time. This allowed us to create a MOFC photoreactor, which effectively combines fluids, photocatalyst and photons for enhanced photocatalytic activity. In doing so we show proof-of-concept for incorporating a wide range of photocatalytic materials within optical fibres for improved product yields, towards more sustainable reactions. We utilised both microfocus X-ray fluorescence and Raman spectroscopy to confirm the MOF had been satisfactorily formed inside the channels of the MOFC, for the first time, validating our material design approach (Figure $1 \&$ S1). Such systems have significant potential as all-in-one photocatalytic reactors. Here we have demonstrated their ability to catalyse organic transformation involving $\mathrm{C}-\mathrm{C}$ bond formation through $\mathrm{C}-\mathrm{H}$ activation. This clearly demonstrates their potential for use in industrially significant processes, utilising sustainable solar energy on a larger scale (Figure S1). The continuous nature of the MOFC approach would be of significant benefit to both pharma and final-chemical industries. Here the selectivity and conversion to be controlled through a wide range of flow-based variables, beyond what is offered from batch processes. Further, the plug-in-and-play, nature of the MOFCs means different systems could be sequentially used to facilitate individual steps of more challenging cascade reactions. This would allow a wide range of processes, to bypass demanding purification procedures for each step, towards in a sustainable photocatalytic for a wide range of chemical processes.

\section{Supporting Information}

Supporting Information is available from the Wiley Online Library or from the author.

\section{Acknowledgements}

MEP, RR, TB and PJAS are grateful to EPSRC for funding (EP/N013883/1, "Adventures in Energy" RR and DJS also acknowledge the EU Horizon 2020 project "MULTI-site organicinorganic HYbrid CATalysts for MULTI-step chemical processes (MULTI2HYCAT)” EU 720783 for funding.

Received: ((will be filled in by the editorial staff))

Revised: ((will be filled in by the editorial staff))

Published online: ((will be filled in by the editorial staff))

\section{References}

[1] G. van Soest, E. Regar, A. F. W. van der Steen, Nature Photonics 2015, 9, 626. 
[2] P. Russell, Science 2003, 299, 358.

[3] Q. Cheng, M. Bahadori, M. Glick, S. Rumley, K. Bergman, Optica 2018, 5, 1354.

[4] A. Amezcua-Correa, J. Yang, C. E. Finlayson, A. C. Peacock, J. R. Hayes, P. J. A.

Sazio, J. J. Baumberg, S. M. Howdle, Adv. Funct. Mater. 2007, 17, 2024.

[5] Optical communications - Corning, https://www.corning.com/optical-

communications/emea/en/home/products/fiber.html, (accessed May 2020).

[6] N. J. Peill, M. R. Hoffman, Environ. Sci. Technol. 1996, 30, 2806.

[7] P. Y. Liou, S. C. Chen, J. C. S. Wu, D. Liu, S. Mackintosh, M. Maroto-Valer, R.

Linforth, Energy. Environ. Sci. 2011, 4, 1487.

[8] P. J. A. Sazio, A. Amezuca-Correa, C. E. Finlayson, J. R. Hayes, T. J. Scheidemantel,

N. F. Baril, B. R. Jackson, D. J. Won, F. Zhang, E. R. Margine, V. Gopalan, V. H. Crespi, J.

V. Badding, Science 2006, 311, 1583.

[9] S. Egusa, Z. Wang, N. Chocat, Z. M. Ruff, A. M. Stolyarov, D. Shemuly, F. Sorin, P. T. Rakich, J. D. Joannopoulos, Y. Fink, Nature Mater. 2010, 9, 643.

[10] M. Pelaeza, N. T. Nolan, S. C. Pillai, M. K. Seery, P. Falaras, A. G. Kontos, P. S. M. Dunlop, J. W. J. Hamilton, J. A. Byrne, K. O'Shea, M. H. Entezari and D. D.Dionysiou, Appl. Catal. B: Environ. 2012, 125, 331.

[11] M. E. Potter, D. J. Stewart, A. E. Oakley, R. P. Boardman, T. Bradley, P. J. A. Sazio, R. Raja, ACS Photonics 2020, 7, 714.

[12] J. Zhang, M. Zhang, C. Yang, X. Wang, Adv. Mater. 2014, 26, 4121.

[13] R. Chen, J. L. Shi, Y. Ma, G. Lin, X. Lang, C. Wang, Angew. Chem. Int. Ed. 2019, 58, 6430.

[14] A. Dhakshinamoorthy, A. M. Asiri, H. Garcia, Angew. Chem. Int. Ed. 2016, 55, 5414.

[15] Y. Chen, D. Wang, X. Deng, Z. Li, Catal. Sci. Technol. 2017, 7, 4893.

[16] X. Yang, X. Jiang, Y. Huang, Z. Guo, L. Shao, Appl. Mater. Inter. 2017, 9, 5590. 
[17] X. Wang, M. Sun, B. Meng, X. Tan, J. Liu, S. Wang, S. Liu, Chem. Commun. 2016, 52, 13448.

[18] Z. Y. Gu, X. P. Yan, Angew. Chem. Int. Ed. 2010, 49, 1477.

[19] J. Qian, F. Sun, L. Qin, Mater. Lett. 2012, 82, 220.

[20] K. Zhou, B. Mousavi, Z. Luo, S. Phatanasri, S. Chaemchuen, F. Verpoort, J. Mater. Chem. A 2017, 5, 952.

[21] A. G. Condie, J. C. González-Gómez, C. R. J. Stephenson, J. Am. Chem. Soc. 2010, 132, 1464.

[22] M. E. Potter, C. P. Ross, D. Gianolio, R. Rios, R. Raja, Catal. Sci. Technol. 2020, 10, 7262.

[23] L. W. Zhang, H. B. Fu, Y. F. Zhu, Adv. Funct. Mater. 2008, 18, 2180.

[24] Z. Öztürk, M. Filez, B. M. Weckhuysen, Chem. Eur. J. 2017, 23, 10915.

[25] J. Yu, H. Yu, B. Cheng, M. Zhou, X. Zhao, J. Mol. Catal. A: Chem. 2006, 253, 112.

[26] C. J. Wu, J. J. Zhong, Q. Y. Meng, T. Lei, X. W. Gao, C. H. Tung, L. Z. Whu, Org. Lett. 2015, 17, 884. 
Optical fiber technology has opened many avenues for tailoring the photocatalytic performance of a range of photoactive materials. This work shows, for the first time, a unique strategy to incorporating a nanoporous framework (Co ZIF-67) within an optical fiber. This reactor improves the efficiency of $\mathrm{C}-\mathrm{C}$ bond formation using visible light, showing clear potential for industrial applications.

M. E. Potter,* D. J. Stewart, K. Ignatyev, T. Bradley, P. J. A. Sazio and R. Raja

Incorporating Metal Organic Frameworks within Microstructured Optical Fibers towards Scalable Photoreactors

ToC figure

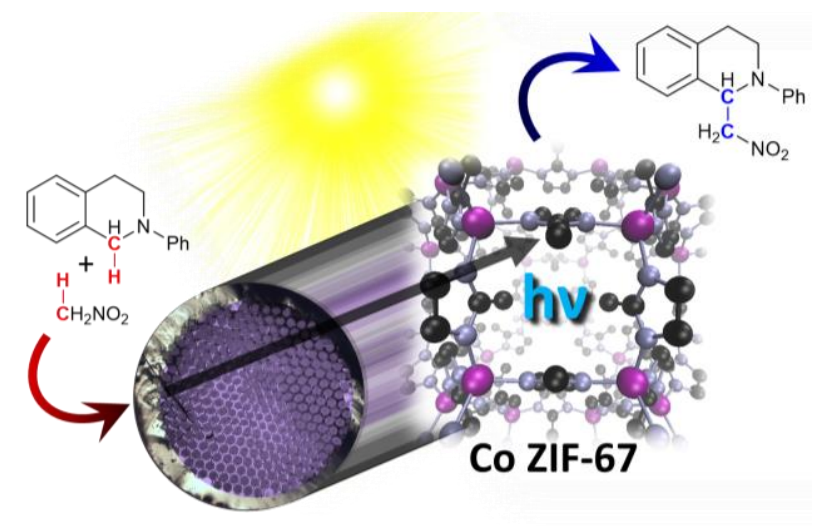


Copyright WILEY-VCH Verlag GmbH \& Co. KGaA, 69469 Weinheim, Germany, 2018.

\author{
Supporting Information
}

Incorporating Metal Organic Frameworks within Microstructured Optical Fibers towards Scalable Photoreactors

Matthew E. Potter, * Daniel J. Stewart, Konstantin Ignatyev, Tom Bradley, Pier J. A. Sazio and Robert Raja

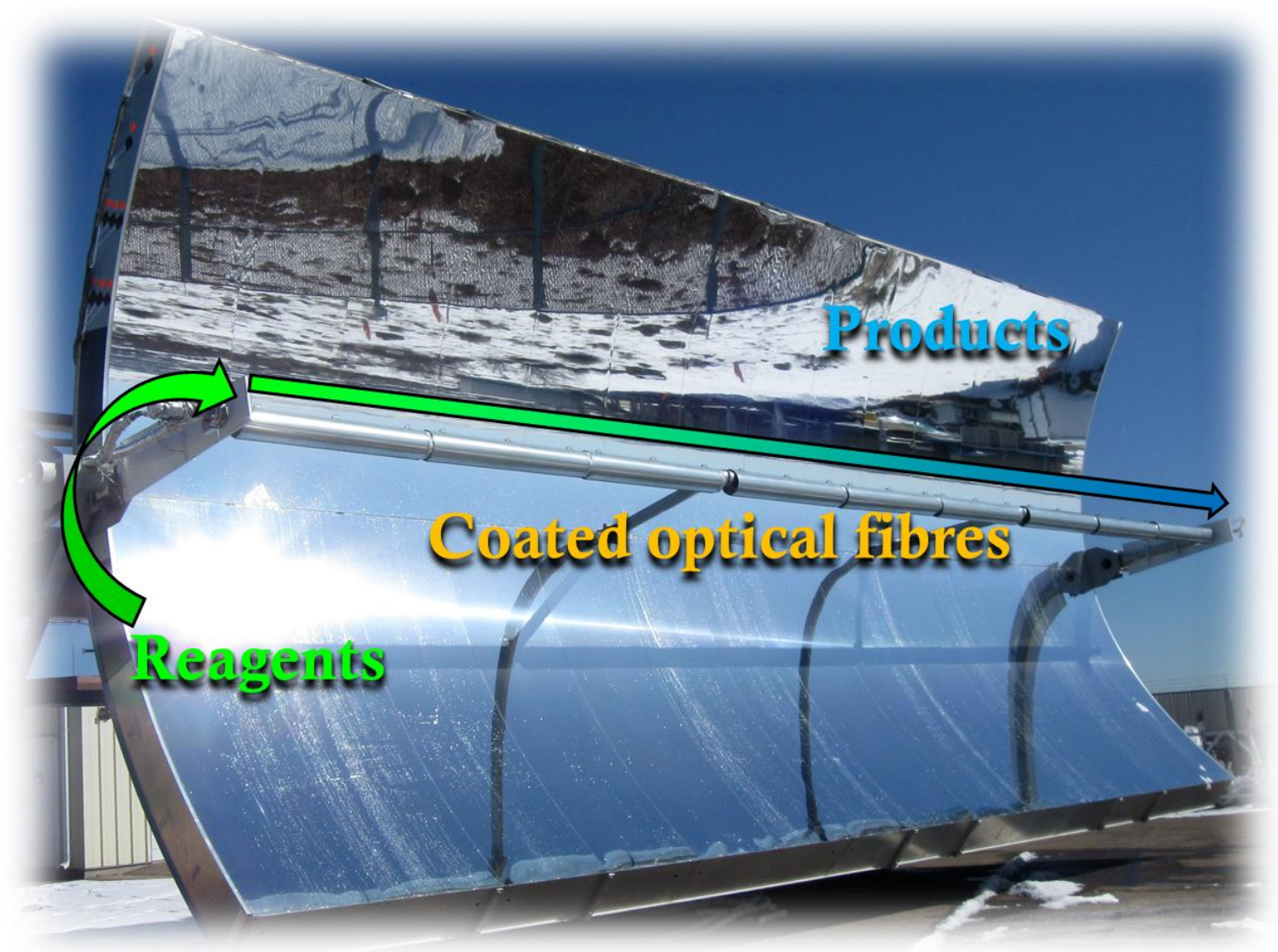

Figure S1: Simplified schematic of our vision for large scale photocatalytic reactors based on optical fibre technology. 


\section{Experimental}

Fibre Synthesis

The 1/8" diameter Kagome lattice optical fibre canes were created using the stack and draw technique $^{[\mathrm{S} 1]}$ within the Silica Fabrication Facility at the Optoelectronics Research Centre at the University of Southampton, as per our previous work. ${ }^{[S 2]}$ The stack and draw techniques begins with fabricating capillaries of the required dimensions with specific aspect ratio. These are then stacked in the desired lattice arrangement. The preform is finally drawn into canes or fibres by adjusting drawing parameters such as feed rate, draw speed and furnace temperature. The cane or fibre dimensions are set according to a simplified mass conservation law, $\mathrm{A}_{\mathrm{p}} \mathrm{v}_{\mathrm{p}}=$ $\mathrm{A}_{\mathrm{f}} \mathrm{V}_{\mathrm{f}}$ where $\mathrm{A}_{\mathrm{p}}$ and $\mathrm{A}_{\mathrm{f}}$ are the cross-sectional area of the preform and cane/fibre respectively while $\mathrm{v}_{\mathrm{p}}$ is the preform feed rate and $\mathrm{v}_{\mathrm{f}}$ the fibre drawing speed. The tower furnace temperature is maintained between the glass softening and melting temperature of around $2100{ }^{\circ} \mathrm{C}$. The very wide viscosity range of pure silica is exploited to ensure precise control of the optical fibres dimensions and uniformity, with online measurement tools such as laser interferometry of the outside diameter.

\section{$\mathrm{TiO}_{2}$ coating}

$\mathrm{TiO}_{2}$ coating was performed on $10 \mathrm{~cm}$ microstructured Kagome lattice canes, with a suspension of $0.1 \mathrm{wt} \%$ commercial $\mathrm{P} 25 \mathrm{TiO}_{2}$ nanopowder (Sigma-Aldrich) in water. The canes were then filled with the suspension using at a rate of $5 \mathrm{~cm} / \mathrm{min}$ using a syringe pump. The suspension was then removed by forcing air through the system, again using a syringe pump, at a speed of $3 \mathrm{~cm} / \mathrm{min}$. This procedure was then repeated from the other end of the cane to achieve an even coating. The sample was subsequently dried overnight at $70{ }^{\circ} \mathrm{C}$, and then heated in an air atmosphere at $400{ }^{\circ} \mathrm{C}$ for $4 \mathrm{hrs}$, at a ramp rate of $5^{\circ} \mathrm{C} / \mathrm{min}$.

\section{Co ZIF-67 coating}


ZIF-67 coating was performed by modifying a procedure by Qian et al. ${ }^{[\mathrm{S} 3]}$ Initially the $10 \mathrm{~cm}$ cane was fully submersed in a stirred solution of $6.18 \mathrm{~g}$ of 2-methyl imidazole in $22 \mathrm{~mL}$ of DI $\mathrm{H}_{2} \mathrm{O}$, to which a solution of $0.61 \mathrm{~g}$ of Cobalt (II) nitrate hexahydrate in $4 \mathrm{~mL}$ of $\mathrm{DI} \mathrm{H}_{2} \mathrm{O}$ was added dropwise. The solution was stirred for 24 hours, when the cane was inverted and was stirred for another 24 hours. The cane was dried, sonicated in ethanol for 15 minutes to remove residual precursors and particles, then dried overnight at $80{ }^{\circ} \mathrm{C}$. This procedure was performed three times to obtain a strong coating. Purple particles were collected from this procedure and confirmed to be Co ZIF-67 through $\mathrm{N}_{2}$ physisorption and X-ray diffraction characterisation.

\section{1) Interior channel of MSOF}

2) Deposit initial $\mathrm{TiO}_{2}$ layer

3)
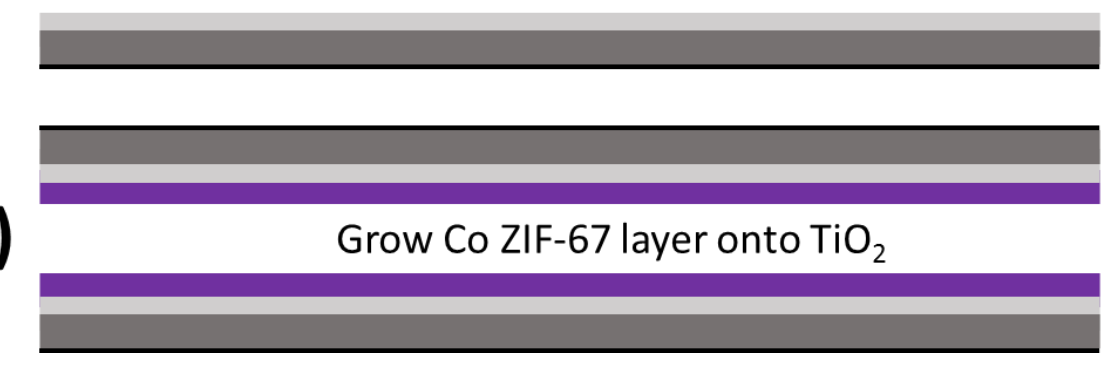

Figure S2: Schematic of our approach to create Co ZIF-67 coated optical fibres (MSOF).

Synthesis of N-phenyl-1,2,3,4-tetrahydroisoquinoline (PhTHIQ)<smiles>c1ccc(N2CCc3ccccc3C2)cc1</smiles> 
PhTHIQ was synthesized by ourselves for this study. The procedure for the starting material used throughout the project was as follows: ${ }^{[\mathrm{S} 4]}$ Copper (I) iodide, (476 mg, $\left.2.50 \mathrm{mmol}\right)$ and potassium phosphate $(10.119 \mathrm{~g}, 47.7 \mathrm{mmol})$ were placed under an inert atmosphere before adding 2-propanol (23.8 mL), ethylene glycol (2.6 mL, $47.6 \mathrm{mmol}), 1,2,3,4-$ tetrahydroisoquinoline $(4.8 \mathrm{~mL}, 35.7 \mathrm{mmol})$ and iodobenzene $(2.7 \mathrm{~mL}, 23.8 \mathrm{mmol})$. The reaction mixture was then heated to $90{ }^{\circ} \mathrm{C}$ and left stirring for 24 hours. Afterwards, it was left to cool to room temperature before adding diethyl ether $(50 \mathrm{~mL})$ and water $(50 \mathrm{~mL})$. The organic layer was extracted by diethyl ether $(2 \times 50 \mathrm{~mL})$ before washing the combined organic phases with brine $(50 \mathrm{~mL})$ and drying over magnesium sulfate. The solvent was removed by rotary evaporation, the dark brown oil was purified by column chromatography on silica gel (hexane/ethyl acetate 19:1). The yield was recorded at $3.238 \mathrm{~g}, 65 \%$. A peach solid with $\mathrm{R}_{\mathrm{f}}$ of 0.25 in the eluent mentioned.

${ }^{1} \mathrm{H}$ NMR (400 MHz, $\left.\mathrm{CDCl}_{3}\right): \delta$ 7.32-7.27 (2H, m), 7.21-7.14 (4H, m), $6.99(2 \mathrm{H}, \mathrm{d}, \mathrm{J}=8.0$ Hz), $6.83(1 \mathrm{H}, \mathrm{t}, \mathrm{J}=7.3 \mathrm{~Hz}), 4.42(2 \mathrm{H}, \mathrm{s}), 3.57(2 \mathrm{H}, \mathrm{t}, \mathrm{J}=5.9 \mathrm{~Hz}), 3.00(2 \mathrm{H}, \mathrm{t}, \mathrm{J}=5.8 \mathrm{~Hz})$ ${ }^{13} \mathrm{C}$ NMR (400 MHz, $\left.\mathrm{CDCl}_{3}\right): \delta 150.71,135.03,134.63,129.34,128.66,126.67,126.47$, $126.16,118.80,115.29,50.89,46.67,29.27$

IR (thin film): $3055 \mathrm{~cm}^{-1}$ (s, sp $\left.\mathrm{sp}^{2} \mathrm{C}-\mathrm{H}\right), 2827 \mathrm{~cm}^{-1}\left(\mathrm{~s}, \mathrm{sp}^{3} \mathrm{C}-\mathrm{H}\right), 1598 \mathrm{~cm}^{-1}(\mathrm{~m}$, benzene $\mathrm{C}=\mathrm{C}$ ), $1501 \mathrm{~cm}^{-1}(\mathrm{~m}$, benzene $\mathrm{C}=\mathrm{C})$

ESI-MS: $m / z, 210$

In agreement with the published literature. ${ }^{[\mathrm{S} 4]}$

Photocatalytic measurements

A stirred solution of $5 \mathrm{~mL}$ Nitromethane and $125 \mathrm{mg}$ of PhTHIQ, with $67.5 \mu \mathrm{L}$ of Mesitylene added as an internal standard, was continuously fed through a $10 \mathrm{~cm}$ section of $1 / 8$ " diameter kagome lattice cane at a rate of $0.36 \mathrm{~mL} / \mathrm{min}$. 


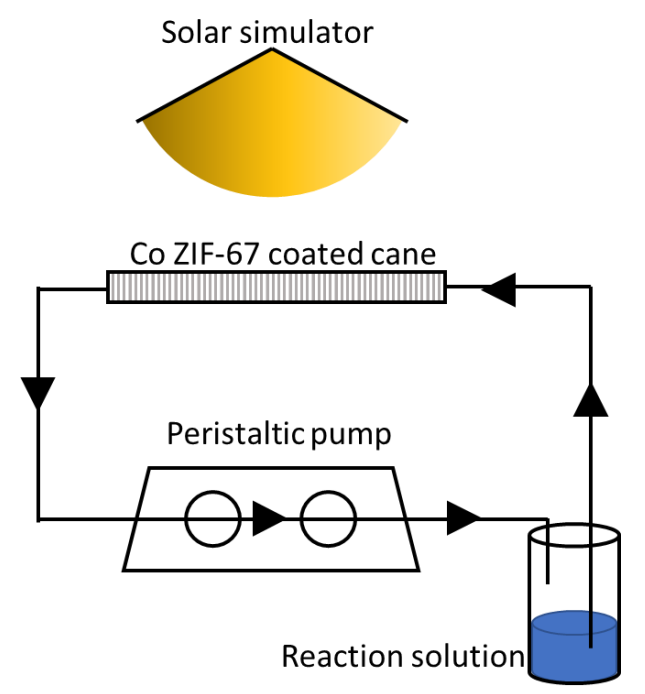

Figure S3: Schematic showing the photocatalytic set-up for the Aza-Henry CDC reaction. The coated cane was approximately $10 \mathrm{~cm}$ away from the output of the solar simulator and positioned at the focal point of a reflective parabolic trough. Apart from the cane, all other components of the rection set-up were shielded from all forms of light. The light source was an Oriel $150 \mathrm{~W}$ Xe short arc lamp, equipped with a AM1.5G solar simulator filter, giving a power output of $50 \mathrm{mWcm}^{-2}$. The mixture was analyzed using ${ }^{1} \mathrm{H}$ NMR, on a Bruker AV400 FT-NMR spectrometer in $\mathrm{CDCl}_{3}$. Chemical shifts for proton and carbon spectra are reported on the delta scale in ppm, referenced to tetramethylsilane (TMS). Conversion and yield were calculated relative to a mesitylene peak $(2.2 \mathrm{ppm}, 9 \mathrm{H})$, and then compared with a starting material (PhTHIQ) peak at $3.00 \mathrm{ppm}(2 \mathrm{H})$ and a product $\left(\mathrm{MeNO}_{2}-\mathrm{PhTHIQ}\right)$ peak at $5.55 \mathrm{ppm}$ $(1 \mathrm{H})$.

All repeated experiments were found to be within $\pm 2 \%$, as such we adopt this as our error for all catalytic measurements.

For powdered samples PhTHIQ $(0.11 \mathrm{mmol})$ and catalyst $(10 \mathrm{wt} \%)$ were stirred in nitromethane $(1 \mathrm{~mL})$, with mesitylene as an internal ${ }^{1} \mathrm{H}$ NMR standard, for $6 \mathrm{~h}$ at $\mathrm{RT}$. The light source was an Oriel $150 \mathrm{~W}$ Xe short arc lamp, equipped with a AM1.5G solar simulator filter, placed $25 \mathrm{~cm}$ away from the reaction mixture, (giving a power output of $50 \mathrm{mWcm}^{-2}$. 
The mixture was analyzed using ${ }^{1} \mathrm{H}$ NMR, on a Bruker AV400 FT-NMR spectrometer in $\mathrm{CDCl}_{3}$. Chemical shifts for proton and carbon spectra are reported on the delta scale in ppm, referenced to tetramethylsilane (TMS). Conversion and yield were calculated relative to a mesitylene peak (2.2 ppm, 9H), and then compared with a starting material (PhTHIQ) peak at $3.00 \mathrm{ppm}(2 \mathrm{H})$ and a product $\left(\mathrm{MeNO}_{2}-\mathrm{PhTHIQ}\right)$ peak at $5.55 \mathrm{ppm}(1 \mathrm{H})$.

\section{Characterisation}

Microfocus X-ray fluorescence (XRF) imaging was performed at beamline I18 at the Diamond Light Source, Didcot, UK via standard access proposal SP17819. XRF maps were used to identify titania rich areas, scanning an area $400 \mu \mathrm{m}$ x $400 \mu \mathrm{m}$.

ICP measurements were performed on powder made from crushed glass canes. These were conducted on a Varian Vista MPX CCD simultaneous axial ICP-OES instrument, based at MEDAC Ltd. Laboratories.

BET $\mathrm{N}_{2}$ adsorption measurements were performed at liquid nitrogen temperature, by first degassing the samples under vacuum. Surface area was determined by nitrogen adsorptiondesorption isotherms using Micromeritics ASAP 2020, calculated using the BET method.

Powder X-ray diffraction was performed on a Bruker AXS D2 Phaser with Cu radiation (wavelength $=1.5406 \AA$ ). The range scanned was dependent on the expected framework, whilst a step of $0.02^{\circ}$ was used for all scans.

Raman analysis was performed at Diamond Light Source, Harwell, UK using an inVia Renishaw Raman microscope with a $785 \mathrm{~nm}$ laser source. 
(DR) UV-Vis measurements were conducted using a Shimadzu 2600 spectrometer with integrated sphere. Reference standard was $\mathrm{BaSO}_{4}$, and spectra were obtaining over a range of 200-800 $\mathrm{nm}$ in steps of $0.2 \mathrm{~nm}$.

TEM images were obtained using a Tecnai 12 with a EMSIS Morada G2 CCD camera and tungsten filament installed, operated at an accelerating voltage of $80 \mathrm{kV}$. Samples were sonicated in ethanol, pipetted onto carbon film-coated, 200 mesh copper TEM grids and allowed to dry in air before imaging. Images were processed using Radius software. Elemental maps were collected using an EDAX Genesis detector.

SEM images were collected on a JEOL JSM-6500F fitted with a field-emission gun. Images were collected with an accelerating voltage of $20 \mathrm{kV}$. Samples of cane were cut using a zirconia saw and mounted using carbon tape.

Cane characterisation

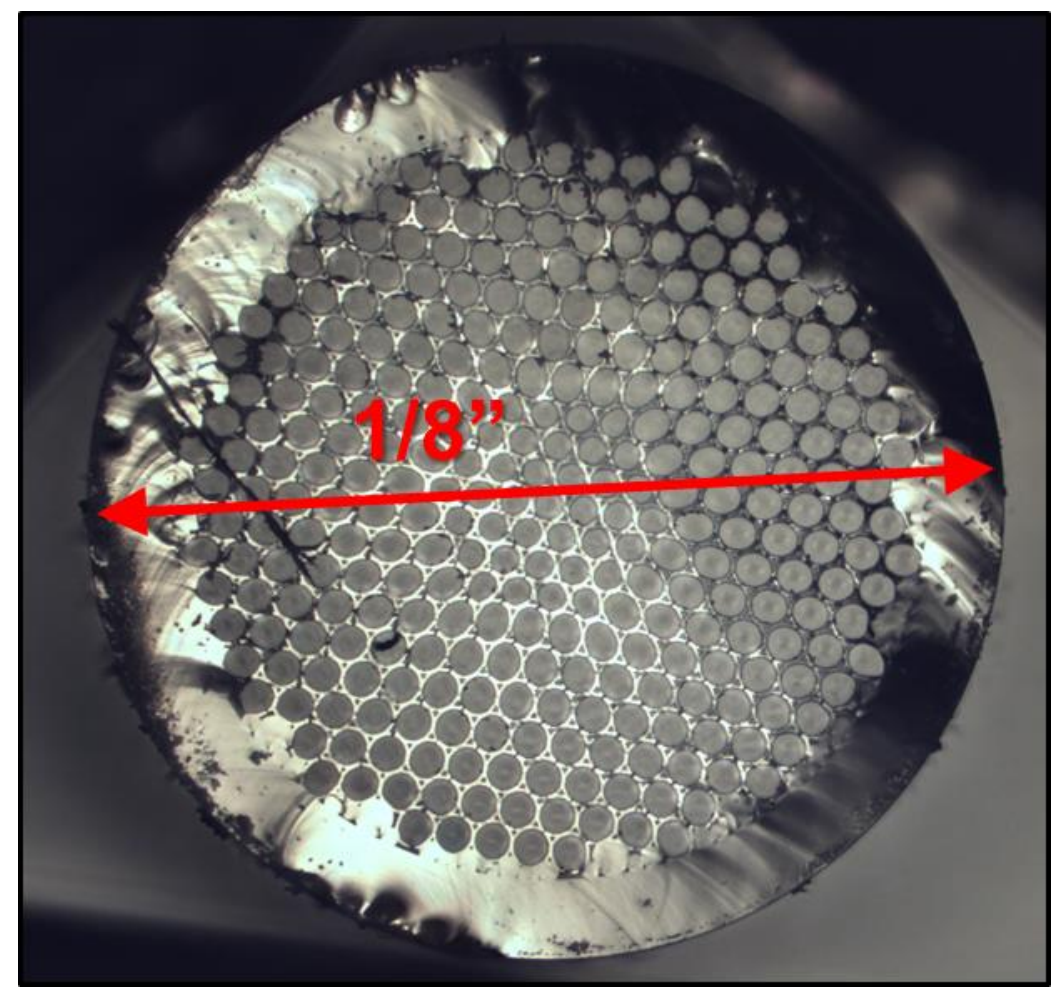


Figure S4: Microscope image showing the internal Kagome lattice structure.

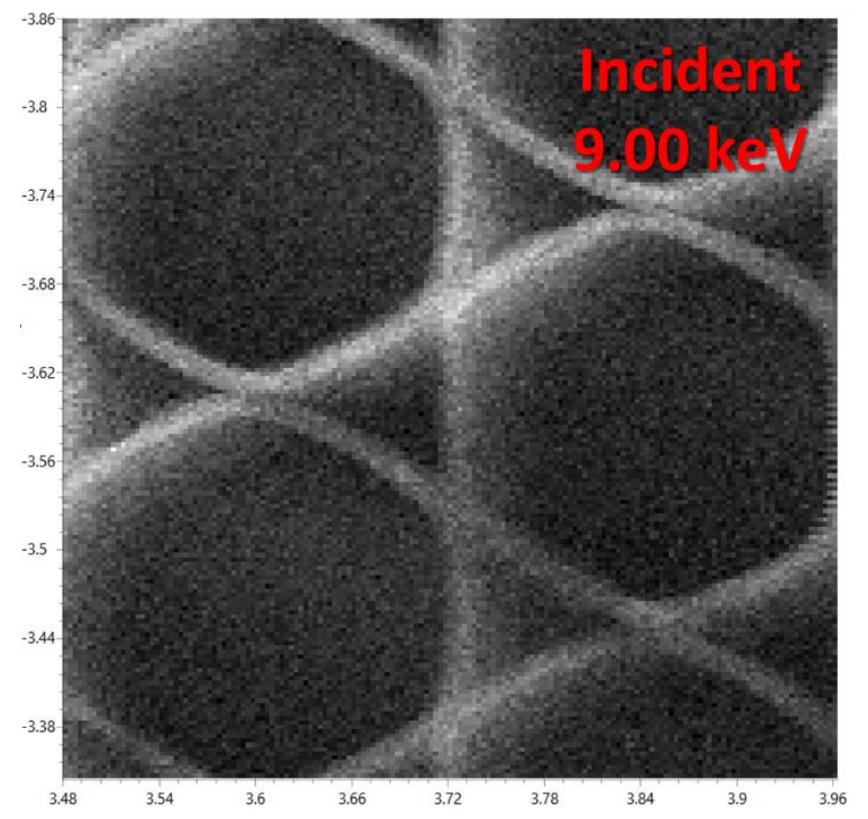

Figure S5: X-ray fluorescence mapping showing the elastically scattered photons from the incident energy $(9.00 \mathrm{keV})$ for the same areas in Figures $2 \mathrm{~A}$ and $2 \mathrm{~B}$ in the main text. Here we highlight the Kagome lattice structure of the cane. Axes are in $\mathrm{mm}$.

ICP analysis

Table S1: ICP analysis showing the evolution of our design process.

\begin{tabular}{llll}
\hline Element & $\mathrm{Si}(\mathrm{wt} \%)$ & $\mathrm{Ti}(\mathrm{wt} \%)$ & $\mathrm{Co}(\mathrm{wt} \%)$ \\
\hline Bare Cane & 41.78 & $<0.001$ & $<0.001$ \\
$\mathrm{TiO}_{2}$ loaded cane & 41.83 & 0.013 & $<0.001$ \\
$\mathrm{Co}$ ZIF-67 loaded cane & 40.90 & 0.007 & 0.069 \\
\hline
\end{tabular}

Physicochemical characterisation of powdered samples 


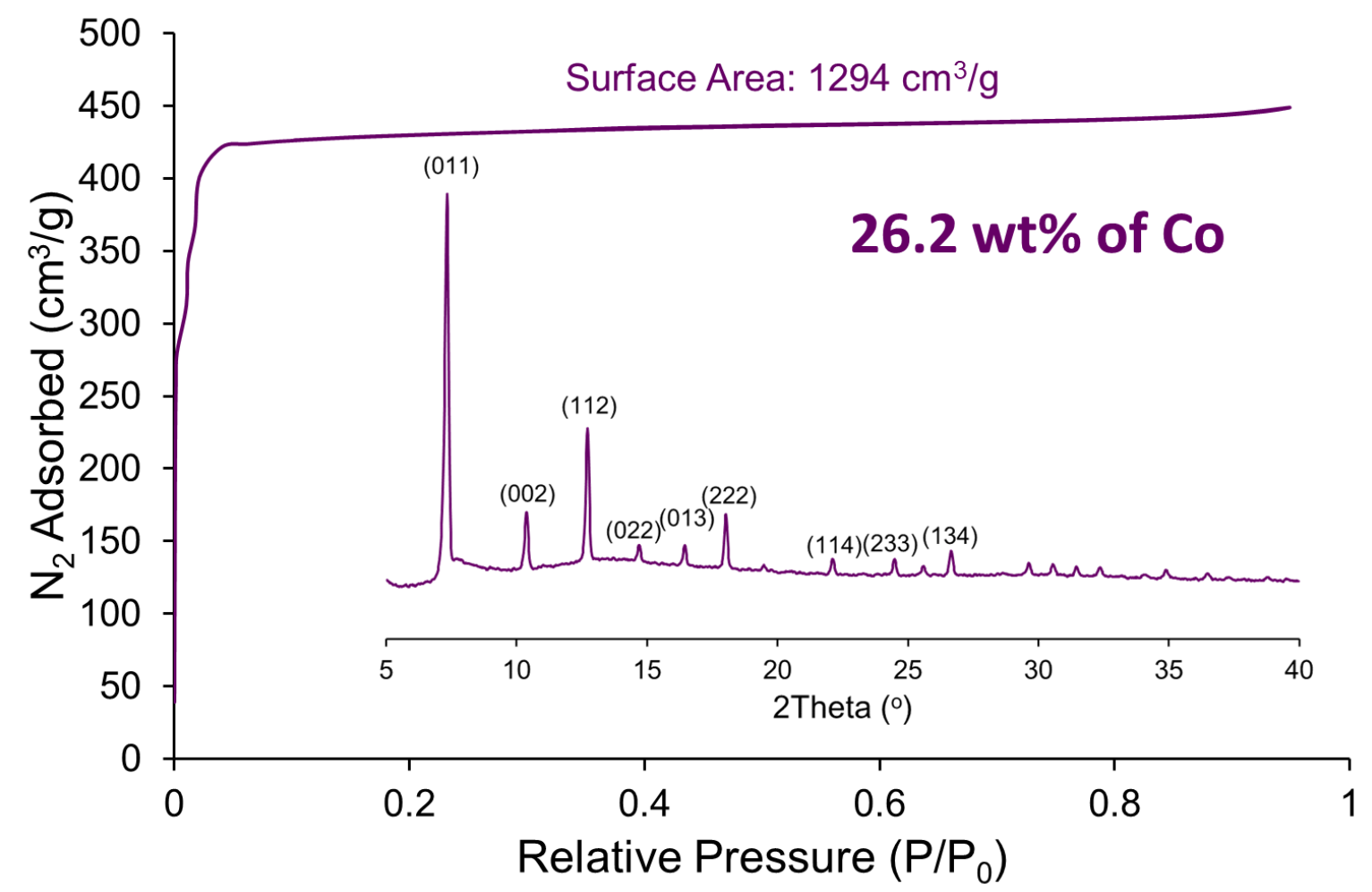

Figure S6: A summary of physicochemical characterisation, confirming the integrity of the Co ZIF-67 particles formed as part of the coating. This included the expected porosity of the powdered Co ZIF-67 and surface area. The inset shows the phase-pure powder X-ray diffraction pattern for the powdered system. ICP analysis confirmed the presence of $26.2 \mathrm{wt} \%$ of Cobalt, in good agreement with the theoretical value of $26.7 \mathrm{wt} \%$.

Cane Raman microscopy images

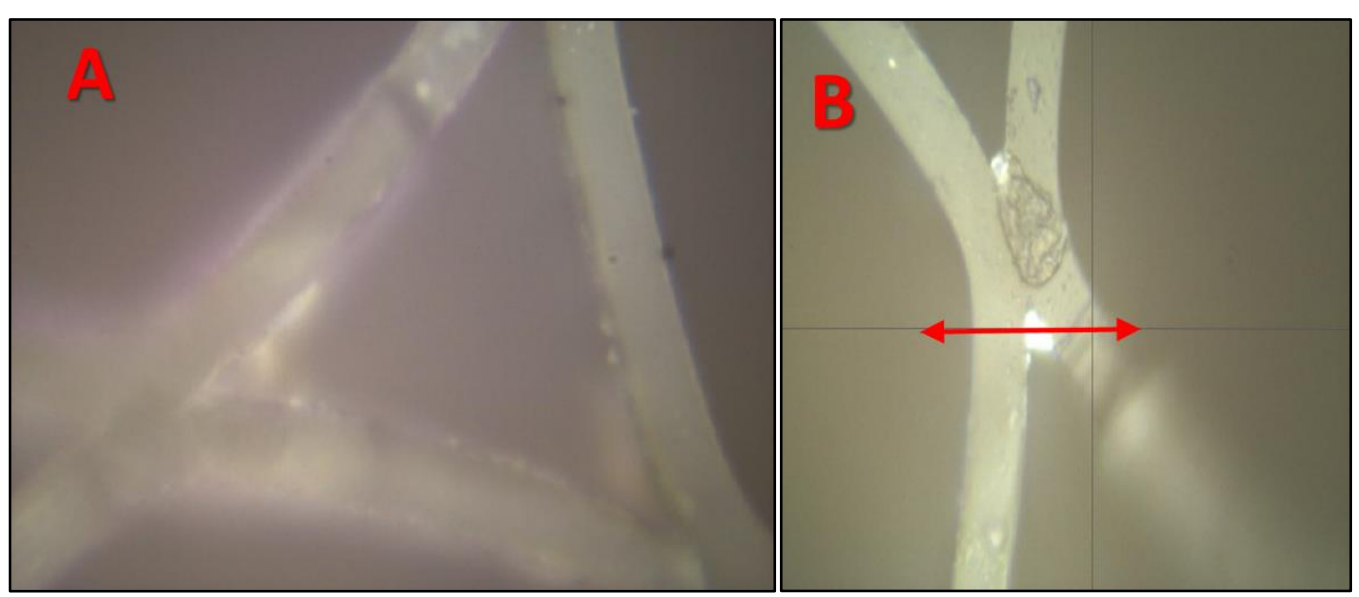



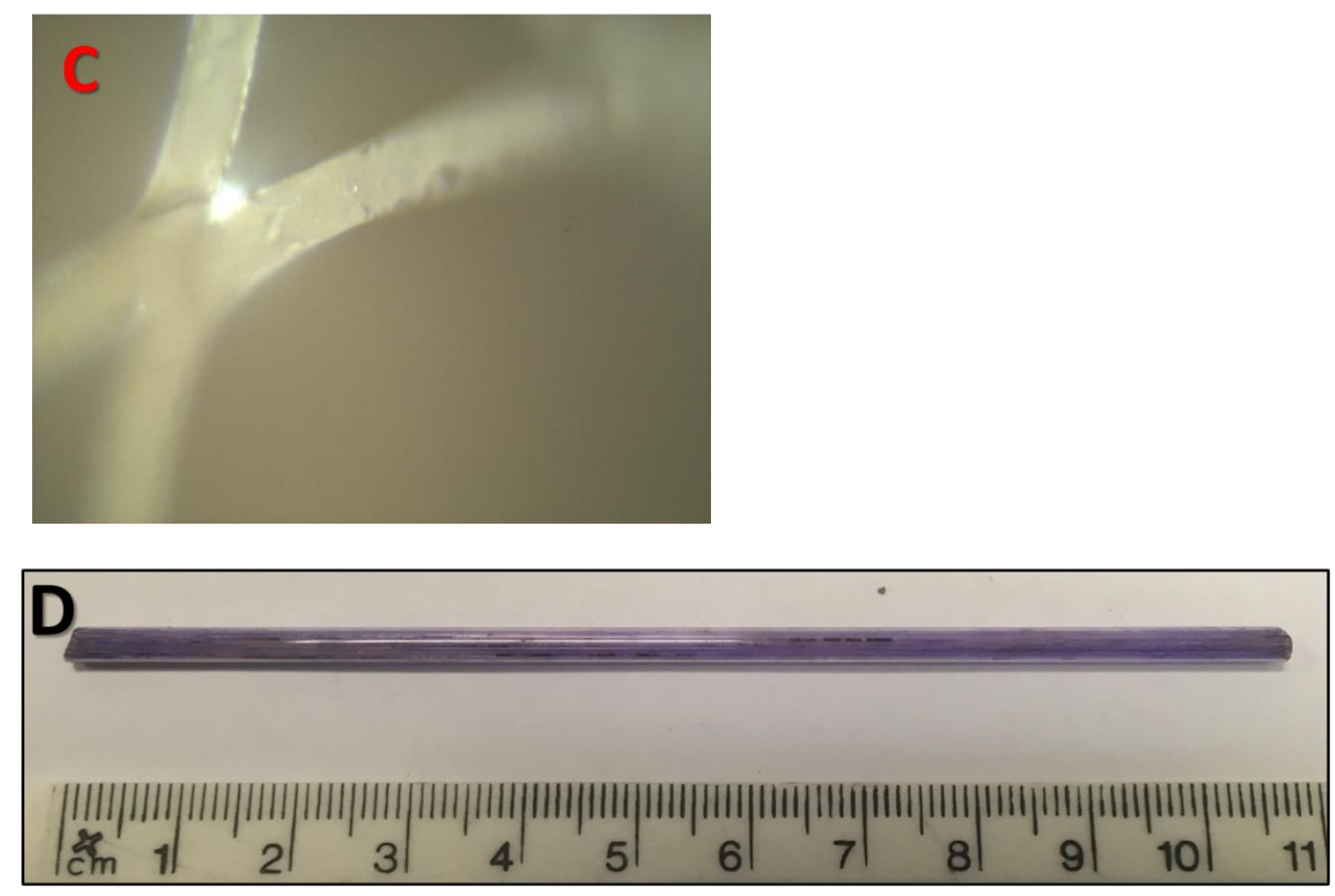

Figure S7: Microscope images showing the coatings of A) Triangular capillary of a Co ZIF67 fibre and B) Image, and path, used for Raman mapping, giving rise to the spectra in Figure 4A, for the Co ZIF-67 coated canes. C) $0.1 \mathrm{wt} \% \mathrm{TiO}_{2}$ coated canes and D) ) Co ZIF-67 coated fibre showing the coating. 


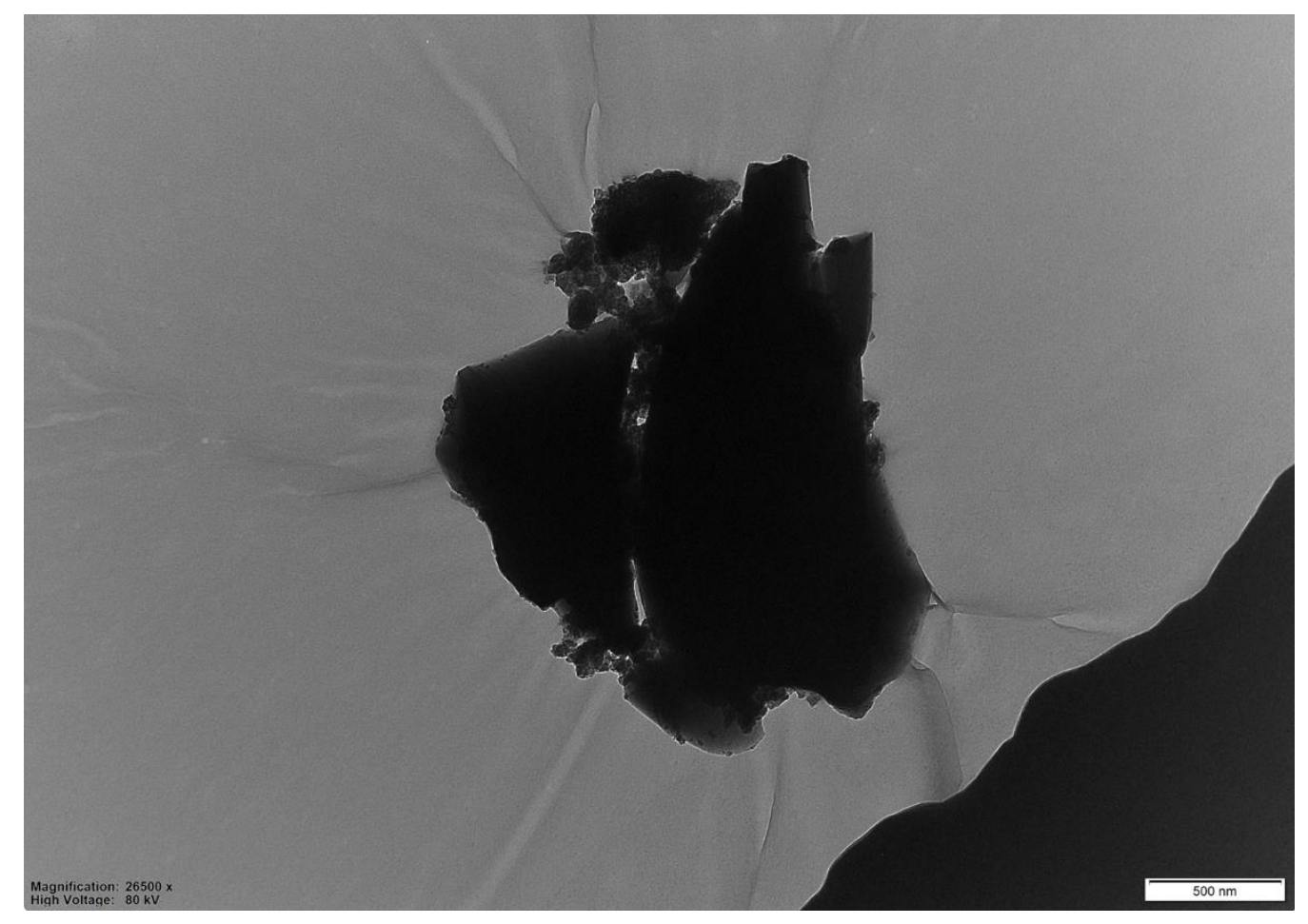

Figure S8: TEM image showing a fragment of coated MOFC.

Literature Co ZIF-67 Raman spectra

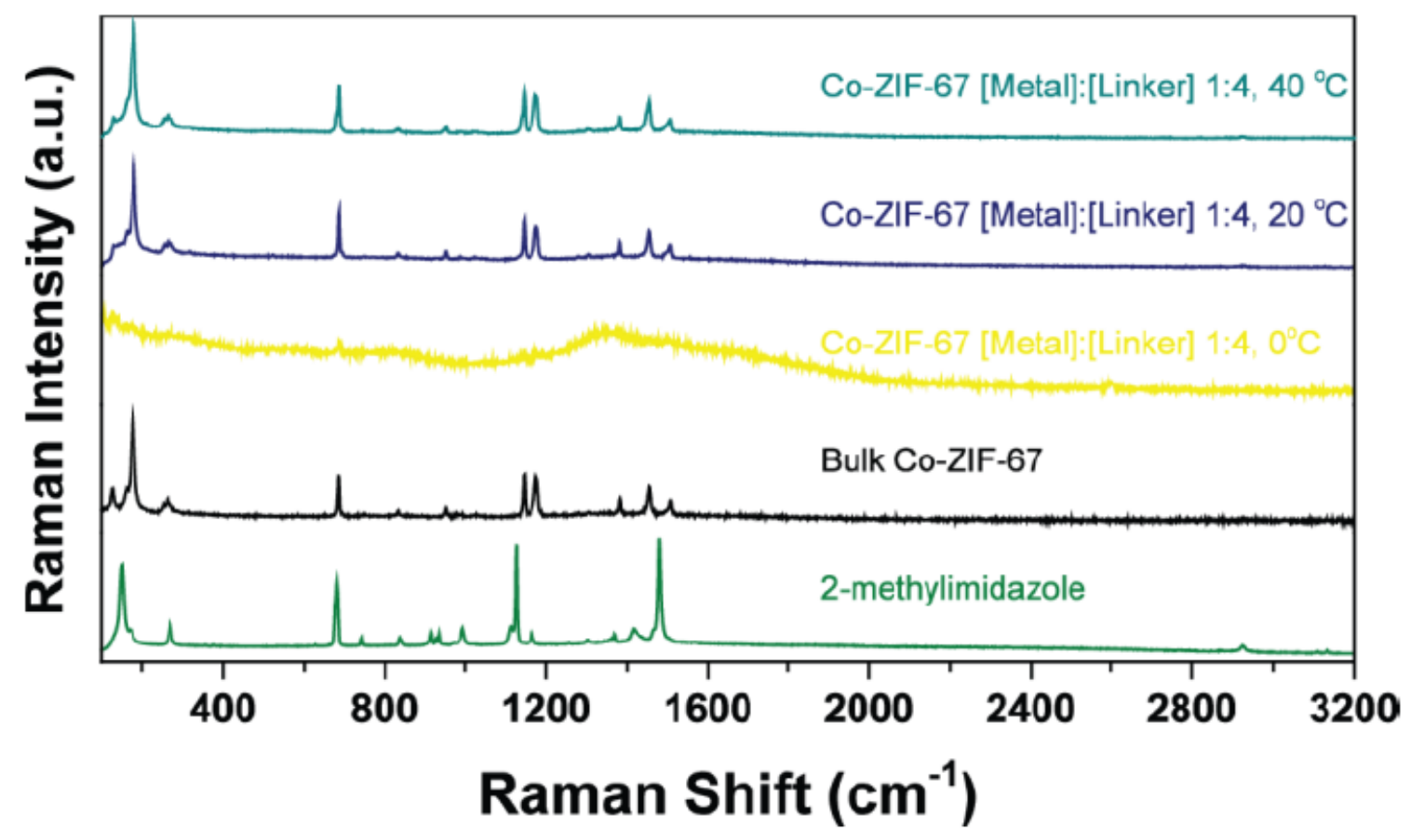

Figure S9: Raman spectra of Bulk Co ZIF-67, as published in the ESI of reference S5 by Weckhuysen et al. 
Catalysis, turnover and quantum yield calculations

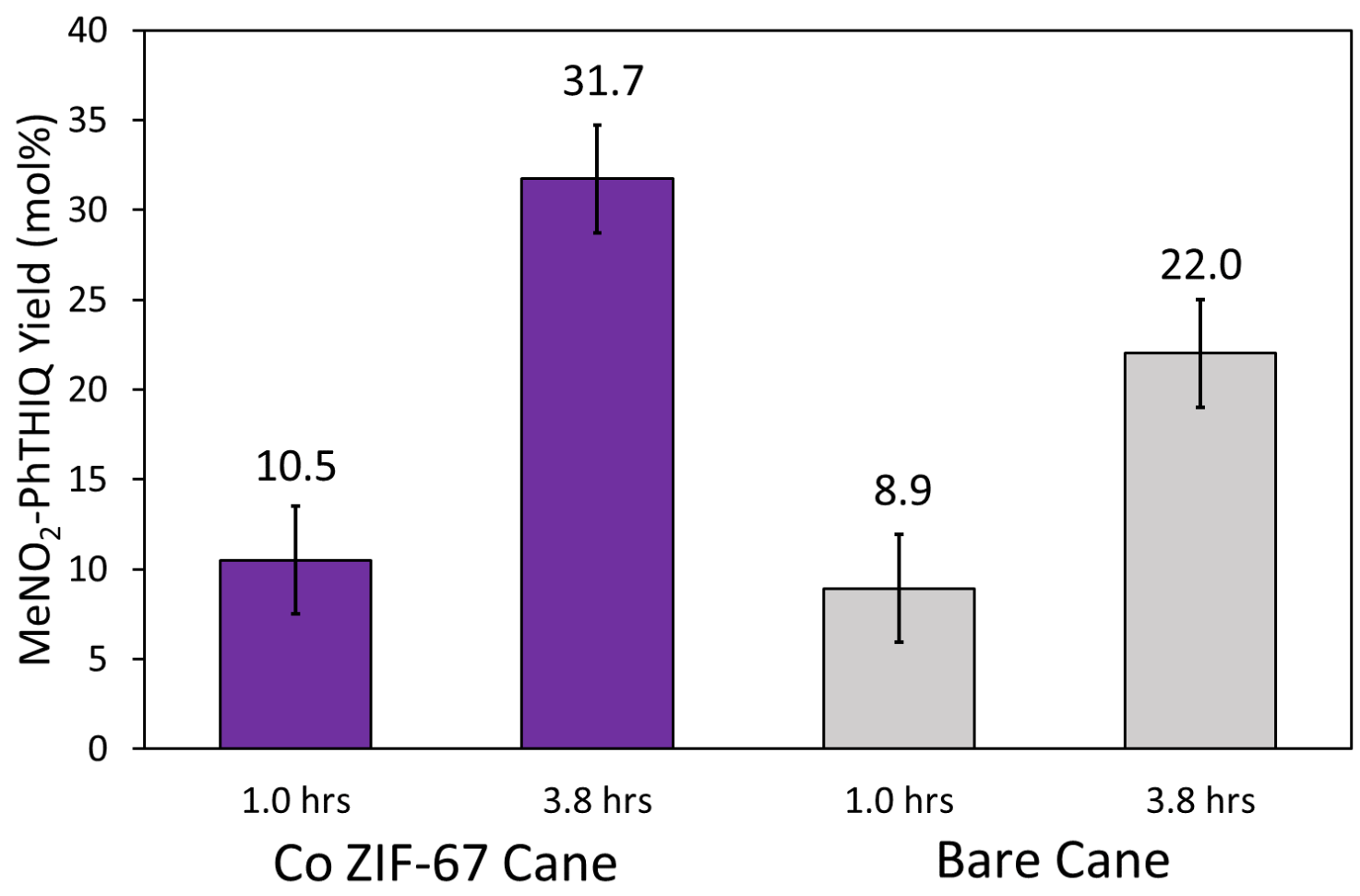

Figure S10: Photocatalytic data comparing the Co ZIF-67 cane, and the bare cane for the the Aza-Henry CDC reaction using Co ZIF-67. Conditions: 5 mL MeNO 2,125 mg of PhTHIQ, $67.5 \mu \mathrm{L}$ of mesitylene standard, flowing at $0.36 \mathrm{~mL} / \mathrm{min}$, looped reactant solution. Errors are estimated as $\pm 3 \mathrm{~mol} \%$.

TON and TOF calculations for Co ZIF-67 MOFC

We used to $10 \mathrm{~cm}$ canes which weighed $0.773 \mathrm{~g}$ in total. ICP (Table S1) shows $0.07 \mathrm{wt} \%$ of this was cobalt, which is a total mass of $0.541 \mathrm{mg}$ of cobalt and $9.182 \times 10^{-3} \mathrm{mmol} .125 \mathrm{mg}$ of PhTHIQ was used, corresponding to $0.598 \mathrm{mmol}$. Conversions of 31.7 and $10.5 \mathrm{~mol} \%$, gave TONs of 20.8 and 6.8 respectively.

The internal volume of the cane $(1 / 8$ " diameter and $10 \mathrm{~cm}$ length) gives a maximum volume of $0.79 \mathrm{~mL}$. The total reaction volume was $5 \mathrm{~mL}$, therefore for every hour of reaction 
(pumping), the materials is only in contact with the catalyst for $0.79 / 5 \mathrm{~mL}=0.158 \mathrm{hr}$. Hence 6 and 24 hours of 'reaction time', corresponds to a contact time of 0.95 and $3.80 \mathrm{hrs}$ respectively. This gives TOFs of 7.2 and $5.4 \mathbf{h r}^{-1}$.

Quantum yield for Co ZIF-67 MOFC

Our fibre is $10 \mathrm{~cm}$ in length, $0.3175 \mathrm{~cm}$ in diameter. We assume, due to the influence of the parabolic trough, that the surface area of $(10 \mathrm{~cm} \times \pi \times 0.3175 \mathrm{~cm}) \underline{9.97 \mathrm{~cm}^{2}}$ is constantly exposed to the light during the reaction.

The lamp has an output of $50 \mathrm{~mW} / \mathrm{cm}^{2}$, therefore the total power of photons adsorbed by the external surface area is $\left(50 \mathrm{~mW} / \mathrm{cm}^{2} \times 9.97 \mathrm{~cm}^{2}\right) \underline{498.7 \mathrm{~mW}}$ or $\underline{0.4987 \mathrm{~J} / \mathrm{s}}$.

The lamp ran for 6 hours, so the total power the reactor was exposed to is $(6 \mathrm{hr} \times 0.4987 \mathrm{~J} / \mathrm{s} \mathrm{x}$ $3600 \mathrm{~s} / \mathrm{hr}) \underline{10773 \mathrm{~J}}$.

The lamp is a solar simulator, with an array of wavelengths. We approximate, in both cases, that the wavelength of the light is $550 \mathrm{~nm}$. A $550 \mathrm{~nm}$ photon has an energy of $\left(6.626 \times 10^{-34} \mathrm{Js}\right.$ x $\left.2.998 \times 10^{8} \mathrm{~m} / \mathrm{s} / 550 \times 10^{-9} \mathrm{~m}\right) \underline{3.61 \times 10^{-19} \mathrm{~J}}$.

This means that the total number of photons the reactor was exposed to is $\left(10773 \mathrm{~J} / 3.61 \times 10^{-}\right.$ $\left.{ }^{19} \mathrm{~J}\right) \underline{2.98 \times 10^{22}}$. This is therefore $\left(2.98 \times 10^{22} / 6.023 \times 10^{23} / \mathrm{mol}\right) \underline{0.0495 \mathrm{~mol}}$ of photons. We converted $10.5 \mathrm{~mol} \%$ of $0.125 \mathrm{~g}$ of PhTHIQ, which has a molar mass of $209.3 \mathrm{~g} / \mathrm{mol}$. Therefore we converted $(0.105 \times 0.125 \mathrm{~g} / 290.3 \mathrm{~g} / \mathrm{mol}) \underline{6.271 \times 10^{-5} \mathrm{~mol}}$ of PhTHIQ. This is believed to be a 1 photon reaction, therefore the moles of photons used was also $\underline{6.271 \times 10^{-5}}$ $\underline{\text { mol. }}$

Therefore we estimate the quantum yield is $\underline{\mathbf{0 . 1 3} \%}\left(100 \times 6.271 \times 10^{-5} \mathrm{~mol} / 0.0495 \mathrm{~mol}\right)$. This is a lower limit, as we have assumed all photons that were in contact with the reactor were absorbed. Because of this it is possible that the quantum yield is under-estimated by an order of magnitude. 
TON and TOF calculations for Co ZIF-67 Powder

Catalytic conditions for powdered Co ZIF-67 were: PhTHIQ $(0.11 \mathrm{mmol})$ and catalyst (10 wt $\%)$ were stirred in nitromethane $(1 \mathrm{~mL})$, with mesitylene as an internal standard for 6 hours. The light source was an Oriel $150 \mathrm{~W}$ Xe short arc lamp, equipped with an AM1.5G solar simulator filter. For further details see our previous work.

$0.11 \mathrm{mmol}$ of PhTHIQ at $10 \mathrm{wt} \%$ catalyst corresponds to $2.3 \mathrm{mg}$ of Co ZIF-67, which contains $0.0104 \mathrm{mmol}$ of Cobalt. We achieved conversions of $82 \mathrm{~mol} \%$, giving a TON of 8.7. Over 6 hours of contact time, this gave a TOF of $1.4 \mathbf{h r}^{-1}$.

Quantum yield for Co ZIF-67 Powder

Our solution is estimated as a cylinder with a length of $2.3 \mathrm{~cm}$ and a diameter of $1.2 \mathrm{~cm}$. We therefore estimate the exposed surface area is the top of the solution $(\pi \times 0.6 \mathrm{~cm} \times 0.6 \mathrm{~cm})$ $\underline{1.13 \mathrm{~cm}^{2}}$ plus the curved surface of the cylinder $(2.3 \mathrm{~cm} \times \pi \times 1.2 \mathrm{~cm}) \underline{8.67 \mathrm{~cm}^{2}}$. Therefore the total exposed area is $\left(8.67 \mathrm{~cm}^{2}+1.13 \mathrm{~cm}^{2}\right) \underline{9.80 \mathrm{~cm}^{2}}$. Again, we assume this is constantly exposed to the light source, due to reflective foil throughout the reaction.

The lamp has an output of $50 \mathrm{~mW} / \mathrm{cm}^{2}$, therefore the total power of photons adsorbed by the external surface area is $\left(50 \mathrm{~mW} / \mathrm{cm}^{2} \mathrm{x} 9.80 \mathrm{~cm}^{2}\right) \underline{490.1 \mathrm{~mW}}$ or $\underline{0.4901 \mathrm{~J} / \mathrm{s}}$.

The lamp ran for 6 hours, so the total power the reactor was exposed to is $(6 \mathrm{hr} \times 0.4901 \mathrm{~J} / \mathrm{s} \mathrm{x}$ $3600 \mathrm{~s} / \mathrm{hr}) \underline{10586 \mathrm{~J}}$.

The lamp is a solar simulator, with an array of wavelengths. We approximate, in both cases, that the wavelength of the light is $550 \mathrm{~nm}$. A $550 \mathrm{~nm}$ photon has an energy of $\left(6.626 \times 10^{-34} \mathrm{Js}\right.$ x $\left.2.998 \times 10^{8} \mathrm{~m} / \mathrm{s} / 550 \times 10^{-9} \mathrm{~m}\right) \underline{3.61 \times 10^{-19} \mathrm{~J}}$.

This means that the total number of photons the reactor was exposed to is (10773 $\mathrm{J} / 3.61 \times 10^{-}$ $\left.{ }^{19} \mathrm{~J}\right) \underline{2.93 \times 10^{22}}$. This is therefore $\left(2.93 \times 10^{22} / 6.023 \times 10^{23} / \mathrm{mol}\right) \underline{0.0487 \mathrm{~mol}}$ of photons. 
We converted $82 \mathrm{~mol} \%$ of $0.11 \mathrm{mmol}$ of PhTHIQ. Therefore we converted $\left(0.82 \times 1.10 \times 10^{-4}\right.$ mol) $9.02 \times 10^{-5} \mathrm{~mol}$ of PhTHIQ. This is believed to be a 1 photon reaction, therefore the moles of photons used was also $\underline{9.02 \times 10^{-5} \mathrm{~mol}}$.

Therefore we estimate the quantum yield is $\underline{\mathbf{0 . 1 9} \%}\left(100 \times 9.02 \times 10^{-5} \mathrm{~mol} / 0.0487 \mathrm{~mol}\right)$. This is a lower limit, as we have assumed all photons that were in contact with the reactor were absorbed. Because of this it is possible that the quantum yield is under-estimated by an order of magnitude.

\section{Error estimation}

Our previous work ${ }^{[\mathrm{S6]}}$ on this reaction showed that the errors, based on repeated measurements, on our yields repeated measurements were \pm 3 mol\% of the measured yield. The error on the time of the measurements is miniscule compared to the catalysis analysis, and can be ignored. The minimum detection limit for these ICP measurements was 0.001 $\mathrm{wt} \%$, therefore we suggest this error is also secondary to the catalysis analysis, and can be ignored. Therefore we assume our main source of error in our TOF and TON calculations is the catalysis analysis, we have used this going forward in all error estimation.

For the TON estimation of the powder, varying the $\mathrm{MeNO}_{2}$-PhTHIQ yield by $\pm 3 \mathrm{~mol} \%$ represents an error of 0.32 for the TON, which when divided by the time $(6 \mathrm{hrs})$ for TOF calculation becomes just $\pm 0.05 \mathrm{hr}^{-1}$.

For the TON estimation on the canes, varying the $\mathrm{MeNO}_{2}-\mathrm{PHTHIQ}$ yield by $\pm 3 \mathrm{moL} \%$ represents an error of 1.96 for the TON, which when divided by the time ( $0.95 \mathrm{hrs}$ or $3.8 \mathrm{hrs})$ becomes either $2.06 \mathrm{hr}^{-1}$ or $0.52 \mathrm{hr}^{-1}$.

These errors have been implements where discussing our catalytic data. 
References

S1) J. C. Knight, T. A. Birks, P. St. J. Russell, D. M. Atkin, Optics Lett. 1996, 21, 1547.

S2) M. E. Potter, D. J. Stewart, A. E. Oakley, R. P. Boardman, T. Bradley, P. J. A. Sazio, R.

Raja, ACS Photonics 2020, 7, 714.

S3) J. Qian, F. Sun, L. Qin, Mater. Lett. 2012, 82, 220.

S4) B. L. Korbad, S. H. Lee, Chem. Commun. 2014, 50, 8985.

S5) Z. Öztürk, M. Filez, B. M. Weckhuysen, Chem. Eur. J. 2017, 23, 10915.

S6) M. E. Potter, C. P. Ross, D. Gianolio, R. Rios, R. Raja, Catal. Sci. Technol. 2020, 10, 7262. 